B I O S C I E N C E

J O U R N A L

\title{
INFLUENCE OF DENTAL EROSION ON SHEAR BOND STRENGTH OF CERAMIC BRACKETS BONDED WITH TWO DIFFERENT ADHESIVE SYSTEMS: AN IN VITRO STUDY
}

\author{
Laerte Ribeiro MENEZES-JÚNIOR ${ }^{1}$ (D), Marcos Alan Vieira BITTENCOURT² (i),
} Andomar Bruno Fernandes VILELA ${ }^{3}$ iD, Carlos José SOARES ${ }^{3}$ iD, Diego Figueiredo NÓBREGA 4 iD, Renata Marques de Melo MARINHO ${ }^{(i D}$, Sigmar de Mello RODE ${ }^{6}$ iD, Flávia Pardo Salata NAHSAN ${ }^{7}$, Luiz Renato PARANHOS 8 iD

\footnotetext{
${ }^{1}$ Postgraduate Program in Dentistry, Federal University of Sergipe, Aracaju, Sergipe, Brazil.

2 Department of Pediatric and Community Dentistry, Federal University of Bahia, Salvador, Bahia, Brazil.

${ }^{3}$ Department of Operative Dentistry and Dental Materials, Federal University of Uberlândia, Uberlândia, Minas Gerais, Brazil.

${ }^{4}$ Department of Health Research, University Center CESMAC, Maceió, Alagoas, Brazil.

${ }^{5}$ Department of Dental Materials, Paulista State University, São José dos Campos, São Paulo, Brazil.

${ }^{6}$ Department of Dental Materials and Prosthesis, Paulista State University, São José dos Campos, São Paulo, Brazil.

7 Department of Dentistry, Federal University of Sergipe, Lagarto, Sergipe, Brazil.

${ }^{8}$ Department of Preventive and Community Dentistry, Federal University of Uberlândia, Uberlândia, Minas Gerais, Brazil.
}

Corresponding author:

Marcos Alan Vieira Bittencourt

Email: alan_orto@yahoo.com.br

How to cite: MENEZES-JÚNIOR, L.R., et al. Influence of dental erosion on shear bond strength of ceramic brackets bonded with two different adhesive systems: an in vitro study. Bioscience Journal. 2021, 37, e37005. https://doi.org/10.14393/BJ-v37n0a2021-56246

\begin{abstract}
This study aimed to analyze the shear bond strength (SBS) of ceramic orthodontic brackets bonded with two different adhesive systems to intact and eroded teeth. Ceramic brackets were bonded to 72 bovine central incisors divided into four groups, defined by two study factors: enamel condition (control group, kept in artificial saliva; and experimental group, eroded by using immersion cycles in Coke ${ }^{\mathrm{TM}}$ for 90 seconds, every six hours for five days), and adhesive system type (Transbond ${ }^{\mathrm{TM}}$ XT or Transbond ${ }^{\mathrm{TM}}$ Plus Color Change). Polycrystalline ceramic brackets were adhesively fixed on all specimens using the same light curing protocol. SBS was tested using $0.5 \mathrm{~mm} / \mathrm{min}$ and the failure mode was classified. SBS data was analyzed using two-way ANOVA followed by Tukey test. The adhesive remnant index (ARI) scores were analyzed using Kruskal-Wallis test with Dunn's post-hoc pairwise comparison $(\alpha=0.05)$. Percentages of ARI scores between the groups were compared by Fisher's exact test. Spearman's correlation coefficient was applied to investigate the correlation between ARI scores and SBS values. Only the adhesive system factor had significant effect on SBS $(p=0.014)$, Transbond ${ }^{T M}$ Plus Color Change showing higher values. No significance was found for enamel condition ( $p=0.665)$ or the interaction between adhesive system and enamel condition $(p=0.055)$. ARI scores frequencies differed between groups $(p<0.001)$. The median ARI scores were statistically different for most comparisons among the groups. However, no significant correlation was found between ARI scores and SBS. In conclusion, the type of adhesive system affected the SBS of ceramic brackets to dental enamel, but the enamel condition, intact or eroded, had no significant effect. There was no correlation between ARI scores and SBS values, although eroded enamel tended to retain more adhesive after bracket removal.
\end{abstract}

Keywords: Ceramics. Orthodontic Adhesive. Orthodontic Brackets. Tooth Erosion. 


\section{Introduction}

Bonded brackets are routinely used in fixed orthodontic therapy, therefore being of great importance to achieve adequate bond strength between the bracket and the enamel surface (Cozza et al. 2006). Successful bonding on tooth surface requires preparation of the enamel for proper mechanical bonding, adequate bracket base design, and a proper adhesive (Breuning et al. 2014). Studies have demonstrated possible changes in tooth enamel and dentin resulting from their exposure to erosive agents of the diet, especially through large ingestion of acidic foods and beverages (El Aidi et al. 2010). Dental erosion comprises an acid-induced wear, characterized by initial softening of the enamel surface, followed by continuous layer-by-layer dissolution of the enamel crystals, leading to a permanent loss of tooth volume (Lussi et al. 2011). It is a common problem in modern societies due to the increased consumption of acidic beverages, such as soft drinks, sports drinks, fruit juices, and teas, which present a high potential to provoke dental demineralization (Correr et al. 2009). Although some isolated surveys evaluating the prevalence of dental erosion have been performed in different populations with different ages, it is difficult to establish comparisons between them because of the different samples and evaluation methods applied on each one. However, it is a consensus that there is a rising prevalence of dental erosion among children, adolescents, and adults, as a result of the increased consumption of those acid drinks, added to psychosomatic eating disorders and current lifestyles (Gambon et al. 2012).

Most of the previous studies evaluating the shear bond strength (SBS) of brackets have used conventional stainless steel brackets, a few have studied ceramic brackets (Gittner et al. 2010; Santin et al. 2015), and no published article has evaluated the SBS of ceramic brackets bonded to eroded teeth. Stainless steel brackets are functionally faultless but they have esthetic disadvantages (Gittner et al. 2010). The increasing demand for esthetics has led to a significant increase in the demand for and use of ceramic brackets. Most ceramic brackets are produced from aluminum oxide (alumina) particles, being available in polycrystalline and monocrystalline forms, according to the manufacturing process (Elekdag-Turk and Ebulkbash 2018). The most evident difference between polycrystalline and monocrystalline brackets is the translucency, being the monocrystalline brackets, also referred to as sapphire brackets, clearer, although in vitro studies have shown that both undergo color changes when subjected to coffee, black tea, coke, and red wine (Oliveira et al. 2014; Guignone et al. 2015). The production process of monocrystalline ceramic brackets is more expensive, while the polycrystalline brackets are simpler to produce and used more frequently (Elekdag-Turk and Ebulkbash 2018).

The excess of adhesive material is a clinical challenge when bonding ceramic brackets, because it may increase the retention of dental plaque, increasing the occurrence of gingivitis and caries (Naranjo et al. 2006). Removing the remnant adhesive material around the bracket is highly recommended but not easy due to the similarity between the color of resins, ceramic brackets, and tooth enamel. Colored orthodontic adhesive systems, which change the color posteriorly during polymerization process, have been created to facilitate this procedure (Türkkahraman et al. 2010). Additionally, some orthodontic adhesive systems release fluoride to the oral environment (Tzou and Darrell 2007; Eissaa et al. 2013), being a means of fluoride delivery adjacent to bracket-enamel interface. When fluoride ions are incorporated into the enamel surface, it forms a fluoroapatite crystal structure that has lower solubility in the oral environment compared with hydroxyapatite, being able to interfere on the ions transferring caused by erosion process, minimizing its effect (Eissaa et al. 2013).

Teeth with eroded surfaces are often seen in clinical practice and sometimes requiring orthodontic procedures. Although adhesive restorations on eroded enamel exhibit lower tensile bond strength and increased susceptibility to microleakage (Casas-Apayco et al. 2014), literature is lacking in studies addressing the bond strength of ceramic orthodontic brackets on eroded teeth. Then, this study was designed aiming to assess, in vitro, the SBS of ceramic brackets bonded, using two different adhesive systems, to teeth subjected to erosive simulation. The null hypotheses were that 1) the enamel condition, intact or eroded, would present similar SBS values, and 2) the orthodontic adhesive system would not influence the SBS values. 


\section{Material and Methods}

This in vitro study was carried out using 72 bovine permanent central incisors. The sample size was based on the study of Attin et al. (2012). Aiming to detect a deviation of 3.00, with significance level set at $5 \%$ and power at $80 \%, 16$ teeth were required in each group. Considering occasional losses, 18 teeth per group were used, resulting in a total of 72 teeth. Four additional teeth were included to verify possible structure changes on enamel surface after the erosive experience compared to the non-eroded enamel. Criteria for tooth selection included teeth with similar age, and no cracks or fractures.

Square pieces of bovine enamel $(4 \mathrm{~mm} \times 4 \mathrm{~mm}$ ) were extracted from the buccal region of the crown using a precision cutting machine (ISOMET Low Speed Saw, Buehler Ltda., Lake Bluff, IL, USA), with a doublesided diamond disc (High Concentration Diamond, Wafering Blade, CT, USA) at constant speed of $300 \mathrm{rpm}$ and cooled with deionized water. Specimens were stored in a glass container with distilled water at room temperature for maintaining the substrate humidity.

A self-cure acrylic resin (Jet ${ }^{\mathrm{TM}}$ Clássico, São Paulo, SP, Brazil) was used for embedding the specimens in PVC rigid rings (Tigre, Joinville, SC, Brazil) maintaining the buccal surface exposed for preparation. Each enamel block was centered and positioned perpendicular to the tube walls by a custom guide. In order to standardize specimens, their surfaces were polished using 320,600, and 1200 silicon carbide grit papers (Norton $^{\mathrm{TM}}$, Guarulhos, SP, Brazil), by using a polisher (PolitrizPolipan ${ }^{\mathrm{TM}}$ 2, São Paulo, SP, Brazil), at high rotation, for 30 seconds (Casas-Apayco et al. 2014).

Specimens were randomly divided into two initial groups, by using an online randomization program (https://www.random.org/), according to the storage media. Artificial saliva was used in control group (Ct) and Coke $^{\mathrm{TM}}$ was used in eroded group $(\mathrm{Er})$ for creating an erosive challenge. The $\mathrm{pH}$ of each solution was measured in triplicate using a calibrated bench $\mathrm{pH}$ meter (Q400AS Quimis ${ }^{\mathrm{TM}}$, Diadema, SP, Brazil). Specimens of Ct were maintained for five days immersed in buffered artificial saliva (Byoformula, São José dos Campos, $\mathrm{SP}, \mathrm{Brazil})$. The erosive challenge was performed by submerging specimens of $\mathrm{Er}$ in Coke ${ }^{\mathrm{TM}}$ for 90 seconds, every six hours for five days (Cheng et al. 2009). Mean pH values were $6.8 \pm 0.3$ for artificial saliva and $2.3 \pm$ 0.1 for Coke $^{\mathrm{TM}}$. After each cycle, samples were rinsed with distilled water, dried using absorbent paper, and then immersed in artificial saliva, maintained at room temperature and changed every 24 hours. After five days of $\mathrm{pH}$ cycling, all samples were maintained in distilled water at room temperature until analyzed. Figure 1 shows the composition of each solution.

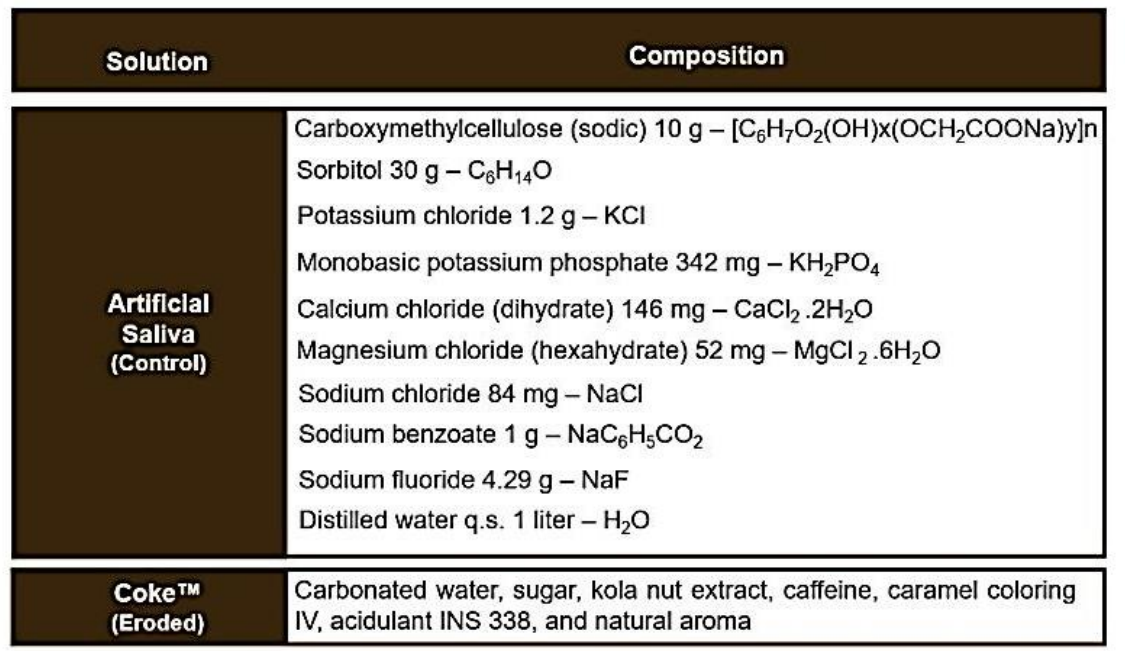

Figure 1. Composition of used solutions, according to manufacturers.

Two specimens of each group were used to verify structure changes on enamel surface after the erosive experience and compare them to the non-eroded enamel by means of a scanning electron microscope (SEM). They were immersed in isopropyl alcohol, inserted in a digital ultrasonic bench (Kondortech model CD-4820, São Carlos, SP, Brazil) for three minutes, air dried and prepared for examination using a SEM (Inspect S50, FEI, Hillsboro, Oregon, USA). Images were captured at $\times 300, x 1000$, and $\times 3000$ magnifications. 
All other specimens were cleaned with an extra-fine fluoride-free pumice slurry (S.S. White, Rio de Janeiro, RJ, Brazil) using a Robinson brush (Microdont, São Paulo, SP, Brazil) for 10 seconds, cleaned with water spray for 15 seconds, and dried with a compressed oil-free stream for 20 seconds. A $37 \%$ phosphoric acid gel (Dentsply, Petrópolis, RJ, Brazil) was applied for etching for 30 seconds, followed by rinsing with a water spray for 30 seconds and air drying for 20 seconds. A liquid primer (Transbond ${ }^{\mathrm{TM}} \mathrm{XT}, 3 \mathrm{M}$ Unitek, Monrovia, CA, USA) was applied to the etched surface and cured for 15 seconds with a light cure unit (Optilight Max, Gnatus, Ribeirão Preto, SP, Brazil).

Polycrystalline ceramic orthodontic brackets of upper right central incisors (Transcend ${ }^{\mathrm{TM}} 3 \mathrm{M}$ Unitek, São José do Rio Preto, SP, Brazil) with mechanical retention base and surface area of $11.78 \mathrm{~mm}^{2}$ were used. Samples of each group were randomly subdivided into two other groups, defined by the adhesive system to be used, Transbond ${ }^{\mathrm{TM}} \mathrm{XT}$ and Transbond ${ }^{\mathrm{TM}}$ Plus Color Change (3M Unitek, Monrovia, CA, USA), then resulting in four groups: CtXT, CtPlus, ErXT and ErPlus. Transbond ${ }^{\mathrm{TM}}$ XT or Transbond ${ }^{\mathrm{TM}}$ Plus Color Change composite resin was applied on the bracket base and, according to the group, placed and pressed on the specimen enamel surface. The excess of composite was removed around the base bracket before it was light cured for 20 seconds using the same light cure unit (Optilight Max, Gnatus, Ribeirão Preto, SP, Brazil), with 5 seconds light incidence over each marginal side of it. The unit tip was positioned closer as possible to the ceramic brackets. All procedures were performed by the same specialist in Orthodontics (LRMJ).

All brackets were debonded using a universal testing machine (EMIC DL-1000, São José dos Pinhais, PR, Brazil). A chisel-edge plunger was mounted in the movable crosshead of the testing machine and positioned closer to the enamel surface to allow a shear loading applied to the enamel-resin interface. A crosshead speed of $0.5 \mathrm{~mm} / \mathrm{min}$ was used, and the maximum force $(\mathrm{N})$ necessary to debond the bracket was recorded. The SBS was calculated by dividing the force values by the base bracket area (MPa).

After debonding, enamel surfaces were evaluated with a stereomicroscope (Carl Zeiss, Goettingen, Germany) at $\times 10$ magnification, by one operator (MAVB) who was blinded to group allocation, to determine the adhesive remnant index (ARI) scores. The order of specimens' analysis for failure classification was randomly performed by using the same online randomization program (www.random.org). The ARI scores were used as a more comprehensive means of defining the sites of bond failure between the enamel, resin, and bracket base: 1 , all the composite remained on enamel surface; 2 , more than $90 \%$ of composite remained on enamel; 3 , more than $10 \%$ but less than $90 \%$ of composite remained on enamel; 4 , less than $10 \%$ of composite remained on enamel; and 5, no composite remained on enamel (Talbot et al. 2000).

The bond strength data were used to test the normality (Kolmogorov-Smirnov test) and homogeneity of variance (Levene test). Two-way analysis of variance (ANOVA) followed by Tukey test were used to compare all groups, involving the enamel condition and the adhesive system used. ARI data were analyzed and Kappa test was applied to verify intra-examiner agreement, showing a high agreement value $(K>0.80)$. Kruskal-Wallis test was used for assessing ARI scores, and Dunn's post-hoc test was performed to correct multiple comparisons of medians between the groups. The percentages of ARI scores between the groups were compared by Fisher's exact test. Spearman's correlation coefficient was also used to investigate the correlation between ARI scores and bond strength values. Significance level was set at $5 \%$. All statistical analyses were performed using the Statistical Software version 5.1 (StatSoft Inc., Tulsa, USA).

\section{Results}

SEM images showed that specimens immersed in artificial saliva did not suffer any erosion, while those immersed in Coke ${ }^{\mathrm{TM}}$ showed significant erosion on the enamel surface (Figure 2).

Mean SBS and standard deviations for all groups are summarized in Table 1. According to the twoway ANOVA and Tukey post-hoc test (Table 2), the adhesive system used influenced significantly the SBS $(p=0.014)$, Transbond ${ }^{\mathrm{TM}}$ Plus Color Change showing higher values. However, no significant influence was found for erosive challenge $(p=0.665)$ neither for interaction between adhesive system and erosive challenge $(p=0.055)$.

ARI score frequencies differed between groups $(p<0.001)$ and are shown in Table 3 . The median ARI scores were statistically different for most comparisons, except when comparing ErXT to ErPlus, and CtXT to CtPlus (Table 4). Spearman's correlation coefficients ranged from weak $(r=0.168, p=0.505)$, between ErXT 
and ErPlus, to strong $(r=-0.449, p=0.062)$, between CtPlus and ErPlus. However, there was no evidence of statistically significant correlations between ARI scores and SBS.

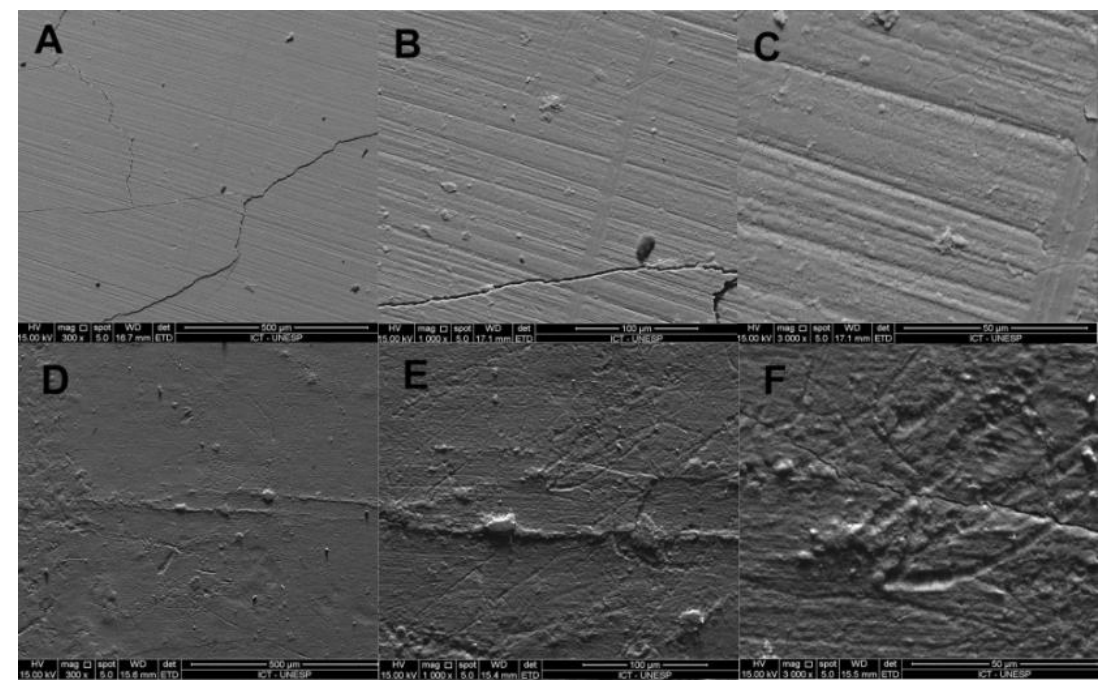

Figure 2. Scanning electron microscopy of enamel surface of specimens of control group $(A, B$, and $C)$ and eroded group after erosive challenge (D, E, and F). A and D, x300; $B$ and $E, x 1000 ; C$ and $F, x 3000$ magnifications. Topographic changes may be observed in images of eroded enamel in comparison to control specimen, which presents only polishing scratches.

Table 1. Mean shear bond strength (MPa) and standard deviation of each studied group.

\begin{tabular}{cc}
\hline Group & Mean \pm SD \\
\hline CtXT & $25.14 \pm 2.88$ \\
ErXT & $26.23 \pm 2.57$ \\
CtPlus & $28.36 \pm 3.52$ \\
ErPlus & $26.64 \pm 3.20$ \\
\hline
\end{tabular}

Table 2. Two-way ANOVA and Tukey post-hoc test for comparing the adhesive systems and used solutions.

\begin{tabular}{cccccc}
\hline Factor & Sum of Squares & $\begin{array}{c}\text { Degrees of } \\
\text { Freedom }\end{array}$ & Mean Square & $\mathrm{F}$ & $\mathrm{p}$ value \\
\hline Adhesive & 59.39 & 1 & 59.39 & 6.33 & $0.014^{*}$ \\
Solution & 1.77 & 1 & 1.77 & 0.19 & 0.665 \\
Adhesive $x$ Solution & 35.72 & 1 & 35.72 & 3.81 & 0.055 \\
Error & 638.03 & 68 & 9.38 & & \\
Total & 734.90 & 71 & & & \\
\hline
\end{tabular}

* Statistically significant.

Table 3. Median and score frequencies of adhesive remnant index (ARI) for each studied group.

\begin{tabular}{cccccccc}
\hline \multirow{2}{*}{ Group } & Median (IQR) & \multicolumn{7}{c}{ ARI score (\%) } & \multirow{2}{*}{ p value $^{\text {a }}$} \\
\cline { 3 - 6 } & & 1 & 2 & 3 & 4 & 5 & \\
\hline CtXT & $3.5(2 ; 4)$ & 5.6 & 27.8 & 16.7 & 27.8 & 22.2 & \\
ErXT & $1.0(1 ; 2)$ & 61.1 & 33.3 & 5.6 & 0.0 & 0.0 & $<0.001$ \\
CtPlus & $3.0(2 ; 3)$ & 0.0 & 27.8 & 50.0 & 16.7 & 5.6 & \\
ErPlus & $2.0(1 ; 2)$ & 38.9 & 44.4 & 16.7 & 0.0 & 0.0 & \\
\hline
\end{tabular}

a Fisher's exact test; IQR - interquartile range. 
Table 4. Dunn's post-hoc test for median comparison and Spearman's correlation coefficients between ARI scores of groups assessed.

\begin{tabular}{ccccccc}
\hline & \multicolumn{2}{c}{ ErPlus } & \multicolumn{2}{c}{ CtPlus } & \multicolumn{2}{c}{ CtXT } \\
\cline { 2 - 6 } & Dunn's & Spearman's & Dunn's & Spearman's & Dunn's & Spearman's \\
\hline \multirow{2}{*}{ CtPlus } & $d=3.34$ & $r=-0.449$ & & & & \\
& $p<0.001$ & $p=0.062$ & & & & \\
CtXT & $d=3.65$ & $r=-0.192$ & $d=-0.31$ & $r=-0.206$ & & \\
& $p<0.001$ & $p=0.445$ & $p=0.377$ & $p=0.412$ & & \\
ErXT & $d=1.04$ & $r=0.168$ & $d=4.38$ & $r=0.215$ & $d=4.69$ & $r=-0.431$ \\
& $p=0.149$ & $p=0.505$ & $p<0.001$ & $p=0.392$ & $p<0.001$ & $p=0.074$ \\
\hline
\end{tabular}

$d-$ Dunn's median differences; $p-p$ values; $r-$ Spearman's correlation coefficients.

\section{Discussion}

The present in vitro study aimed to assess the bond ability of ceramic brackets bonded to teeth subjected to erosive simulation, using two different adhesive systems. The obtained data indicated that the simulated erosive challenge had no significant effect on SBS of brackets to bovine enamel, considering that specimens eroded with Coke $^{\mathrm{TM}}$ presented similar results to those immersed in saliva. Then, the first null hypothesis was accepted. However, SBS was affected by the type of adhesive system, since Transbond ${ }^{\mathrm{TM}}$ Plus Color Change presented SBS values significantly higher than Transbond ${ }^{\mathrm{TM}}$ XT. Therefore, the second null hypothesis was rejected.

Acidic beverages reduce oral cavity $\mathrm{pH}$, and the acidity generates high potential for erosive process, which can modify enamel surface (Ablal et al. 2017). The erosive signs may be assessed qualitatively by SEM analysis (Cheng et al. 2009), but erosion observed using SEM in the present study showed less structural changes on enamel surface after the erosive experience than that observed by other researchers (Owens and Kitchens 2007), probably due to the fact that in most studies the teeth were immersed continuously in the acidic drinks and without artificial saliva use, ignoring the usual oral environment conditions and the possible remineralizing effect of saliva. The irregular areas of samples retrieved when exposed to Coke ${ }^{\mathrm{TM}}$ are observed in different magnifications in Figure 2, with some topographic changes of eroded enamel when compared to control group.

Although some articles report that eroded surfaces present a significant reduced debonding resistance, most studies focused in evaluating the influence of erosive agents in simulated restorative procedures (Casas-Apayco et al. 2014) or during orthodontic treatment, i.e., in brackets previously bonded (Oncag et al. 2005; Sheibaninia et al. 2014). Our results showed that erosion on enamel surface before ceramic bracket placement did not significantly affect bond strength, similar to previous studies performed with metallic brackets (Degrazia et al. 2013; Costenoble et al. 2016). It is not known, however, whether the pumice cleaning or the acid-etch procedures alter all microstructural changes in the enamel imparted by erosive challenge. It may be that Coke ${ }^{\mathrm{TM}}$ does impart some structural changes to the enamel surface, but that these changes are eliminated by the cleaning or the acid-etch procedures. If this were the case, any demineralization imparted by the Coke ${ }^{\mathrm{TM}}$ was eliminated. Another possibility is that erosive challenge alters the enamel surface, but not by an amount sufficient to affect bond strength, regardless of whether the acidetch procedure alters these surface changes.

No significant influence on SBS was also found, in this research, for interaction between adhesive system and erosive challenge. However, when considering solely the adhesive system, significant difference was found, Transbond ${ }^{\mathrm{TM}}$ Plus Color Change showing higher values. Transbond ${ }^{\mathrm{TM}}$ Plus Color Change presents higher Knoop microhardness and degree of conversion when compared to Transbond ${ }^{\mathrm{TM}} \mathrm{XT}$, and these properties are associated with better physical and chemical characteristics as mechanical resistance (Amato et al. 2014). An important fact to point is that, although the manufacturer of Transbond ${ }^{\mathrm{TM}}$ Plus Color Change claims that this adhesive system releases fluoride (Tzou and Darrel 2007), the higher SBS results for Transbond ${ }^{\mathrm{TM}}$ Plus Color Change, in the present study, should not be attributed to the presence of fluoride. The fluoroaluminosilicate in its composition might only be associated with antibacterial properties, inhibiting 
demineralization and increasing remineralization around the bracket, preventing future white spot lesions (Hung et al. 2019). The evidence of this is given by the fact that in the groups bonded with the fluoridated adhesive system, the SBS of the group exposed to the erosive challenge (ErPlus) did not differ from that observed in the control group (CtPlus). Complementary studies are recommended to confirm the releasing of fluoride from the mentioned adhesive system, considering that the information provided by the manufacturer is insufficient.

Ceramic brackets used in the present research showed high SBS values, ranging from around 25 to $28 \mathrm{MPa}$. The physical properties of ceramic materials are the result of their atomic composition as well as ionic and covalent bonds, which give the material hardness, high tensile strength, and a low degree of resistance to fracture and friction (Santin et al. 2015). As deformation of ceramic brackets before fracturing is less than $1 \%$, whereas metal brackets can deform as much as $20 \%$, chemical retentions are used less often than mechanical retentions to avoid undesirable damage to enamel during debonding of orthodontic appliances. However, the Transcend ${ }^{T M}$ ceramic brackets, used in this study, present also chemical retention, and this, according to Wang et al. (1997), may explain our high SBS values. Although increasing the risk of damage tooth enamel during bracket removal, causing problems such as cracks or fractures, no damage was observed, upon microscopic evaluation, in a previous research, even with values over $27 \mathrm{MPa}$ (Sheibaninia et al. 2014).

Additionally, in the present study, there was a high frequency of ARI scores 1 or 2 , and no scores 4 or 5 , in both eroded enamel groups, showing that more than $90 \%$ of bond failure location was at the adhesivebracket interface, with less risk of enamel tear-out. This may be related to the higher apparent irregularity of enamel surface, therefore increasing the bonding area between adhesive system and tooth. Specimens submerged in artificial saliva showed higher prevalence of scores 3, 4, or 5, and half of group CtXT presented indexes 4 or 5 , increasing the risk of enamel fracture when debonding the bracket. Similar to previous study (Linjawi and Abbassy 2016), no significant correlation between ARI scores and SBS was found, although other research have found direct correlation between these parameters, associating high ARI scores with higher SBS values (Faria-Júnior et al. 2015).

Most studies involving enamel alterations and orthodontic adhesive systems are conducted in vitro, although a precise simulation of oral cavity conditions is required for obtaining clinically relevant results, which is hard to achieve. In this study, despite the inherent limitations of an in vitro experimental research, a dynamic model was used aiming to mimic the oral environment using exposure cycles of acid solution alternating with the remineralizing process promoted by artificial saliva. Thus, although the results of the present research should be carefully interpreted, considering that clinical conditions may vary significantly, the bonding process of ceramic orthodontic brackets when enamel is involved in early stages of erosive demineralization, combining different adhesive systems, was highlighted.

\section{Conclusions}

The results of the present study showed that the erosive challenge had no effect on SBS of brackets to dental enamel, considering that the exposure to Coke ${ }^{\mathrm{TM}}$ resulted in similar values to those found in exposure to saliva. However, the adhesive system affected the SBS, Transbond ${ }^{\mathrm{TM}}$ Plus Color Change showing higher values. No significant correlation was found between frequency of ARI scores and SBS values, although eroded enamel tended to retain more adhesive after bracket removal.

\footnotetext{
Authors' Contributions: MENEZES-JÚNIOR, L.R.: conception and design, acquisition of data, analysis of data, drafting the manuscript, writing the manuscript, final approval; BITTENCOURT, M.A.V.: acquisition of data, analysis and interpretation of data, discussing the results, drafting the manuscript, writing and revising the manuscript, final approval; VILELA, A.B.F.: interpretation of data, drafting the manuscript, revising the manuscript, final approval; SOARES, C.J.: interpretation of data, drafting the manuscript, revising the manuscript, final approval; NÓBREGA, D.F.: analysis of data, drafting the manuscript, writing and revising the manuscript, final approval; MARINHO, R.M.M.: acquisition of data, drafting the manuscript, writing and revising the discussion, final approval; RODE, S.M.: acquisition of data, drafting the manuscript, final approval; NAHSAN, F.P.S.: conception and design, analysis and interpretation of data, drafting the manuscript, final approval; PARANHOS, L.R.: conception and design, analysis of data, drafting the manuscript, revising the manuscript, final approval.
}

Conflicts of Interest: The authors declare no conflicts of interest.

Ethics Approval: Not applicable. 
Acknowledgments: The authors would like to thank the funding for the realization of this study provided by the Brazilian agencies FAPITEC/SE (Fundação de Apoio à Pesquisa e à Inovação Tecnológica do Estado de Sergipe - Brasil), through the PROMOB (Academic Mobility Program), Finance Code 1780/2014, CAPES (Coordenação de Aperfeiçoamento de Pessoal de Nível Superior - Brasil), Finance Code 001, and CNPq (Conselho Nacional de Desenvolvimento Científico e Tecnológico - Brasil), Finance Code 307808/2018.

\section{References}

ABLAL, M.A., et al. A novel approach to study in situ enamel erosion and abrasion lesions. Journal of Dentistry. 2017, 59, 78-85.

https://doi.org/10.1016/j.jdent.2017.02.013

AMATO, P.A., et al. Time reduction of light curing: influence on conversion degree and microhardness of orthodontic composites. American Journal of Orthodontics and Dentofacial Orthopedics. 2014, 146(1), 40-46. https://doi.org/10.1016/j.ajodo.2014.03.022

ATTIN, R., et al. Shear bond strength of brackets to demineralize enamel after different pretreatment methods. The Angle Orthodontist. 2012, 82(1), 56-61. https://doi.org/10.2319/012311-48.1

BREUNING, K.H., et al. Bonding metal brackets on tooth surfaces. Dentistry. 2014, 4(5), 231. https://doi.org/10.4172/2161-1122.1000231

CASAS-APAYCO, L.C., et al. Erosive cola-based drinks affect the bonding to enamel surface: an in vitro study. Journal of Applied Oral Science. 2014, 22(5), 434-441. https://doi.org/10.1590/1678-775720130468

CHENG, Z.J., et al. The enamel softening and loss during early erosion studied by AFM, SEM and nanoindentation. Biomedical Materials (Bristol, England). 2009, 4(1), 015020. https://doi.org/10.1088/1748-6041/4/1/015020

CORRER, G.M., et al. Influence of diet and salivary characteristics on the prevalence of dental erosion among 12-year-old schoolchildren. Journal of Dentistry for Children (Chicago, III.). 2009, 76(3), 181-187.

COSTENOBLE, A., et al. Bond strength and interfacial morphology of orthodontic brackets bonded to eroded enamel treated with calcium silicate-sodium phosphate salts or resin infiltration. The Angle Orthodontist. 2016, 86(6), 909-916. https://doi.org/10.2319/111315-764.1

COZZA, P., et al. Shear bond strength of metal brackets on enamel. The Angle Orthodontist. 2006, 76(5), 851-856.

https://doi.org/10.1043/0003-3219(2006)076[0851:SBSOMB]2.0.CO;2

DEGRAZIA, F.W., et al. Influence of dental erosion on the shear bond strength of orthodontic brackets: an in vitro study. Revista da Faculdade de Odontologia - UPF. 2013, 18(1), 83-87. https://doi.org/10.5335/rfo.v18i1.3120

EISSAA, O.E., EL-SHOURBAGY, E.M. and GHOBASHY, S.A. In vivo effect of a fluoride releasing adhesive on inhibition of enamel demineralization around orthodontic brackets. Tanta Dental Journal. 2013, 10(2), 86-96. https://doi.org/10.1016/j.tdj.2013.08.007

EL AIDI, H., et al. Dynamics of tooth erosion in adolescents: a 3-year longitudinal study. Journal of Dentistry. 2010, 38(2), 131-137.

https://doi.org/10.1016/i.jdent.2009.09.012

ELEKDAG-TÜRK, S. and EBULKBASH, H., 2018. Ceramic brackets revisited. In: ASLAN, B.I. and UZUNER, F.D., eds. Current Approaches in Orthodontics, London: IntechOpen, pp. 5-22. Available from: https://doi.org/10.5772/intechopen.79638

FARIA-JÚNIOR, É.M., et al. In-vivo evaluation of the surface roughness and morphology of enamel after bracket removal and polishing by different techniques. American Journal of Orthodontics and Dentofacial Orthopedics. 2015, 147(3), 324-329.

https://doi.org/10.1016/j.ajodo.2014.10.033

GAMBON, D.L., BRAND, H.S. and VEERMAN, E.C.I. Dental erosion in the $21^{\text {st }}$ century: what is happening to nutritional habits and lifestyle in our society? British Dental Journal. 2012, 213(2), 55-57. https://doi.org/10.1038/sj.bdj.2012.613

GITTNER, R., MÜLLER-HARTWICH, R., JOST-BRINKMANN, P.G. Influence of various storage media on shear bond strength and enamel fracture when debonding ceramic brackets: an in vitro study. Seminars in Orthodontics. 2010, 16(1), 49-54. https://doi.org/10.1053/1.sodo.2009.12.004

GUIGNONE, B.C., et al. Color stability of ceramic brackets immersed in potentially staining solutions. Dental Press Journal of Orthodontics. 2015, 20(4), 32-38. https://doi.org/10.1590/2176-9451.20.4.032-038.oar

HUNG, C.Y., et al. Shear bonding strength and thermal cycling effect of fluoride releasable/rechargeable orthodontic adhesive resins containing LiAl-F layered double hydroxide (LDH) filler. Materials (Basel). 2019, 12(19), 3204. https://doi.org/10.3390/ma12193204

LINJAWI, A.I. and ABBASSY, M.A. Comparison of shear bond strength to clinically simulated debonding of orthodontic brackets: an in vitro study. Journal of Orthodontic Science. 2016, 5(1), 25-29. https://doi.org/10.4103/2278-0203.176655

LUSSI, A., et al. Dental erosion: an overview with emphasis on chemical and histopathological aspects. Caries Research. 2011, 45(Suppl 1), 2-12. https://doi.org/10.1159/000325915

NARANJO, A.A., et al. Changes in the subgingival microbiota and periodontal parameters before and 3 months after bracket placement. American Journal of Orthodontics and Dentofacial Orthopedics. 2006, 130(3), 275.e17-275.e22. https://doi.org/10.1016/j.ajodo.2005.10.022

OLIVEIRA, C.B., et al. In vitro study of color stability of polycrystalline and monocrystalline ceramic brackets. Dental Press Journal of Orthodontics. 2014, 19(4), 114-121. https://doi.org/10.1590/2176-9451.19.4.114-121.oar

ONCAG, G., TUNCER, A.V. and TOSUN, Y.S. Acid soft drinks effects on the shear bond strength of orthodontic brackets and a scanning electron microscopy evaluation of the enamel. The Angle Orthodontist. 2005, 75(2), 247-253. https://doi.org/10.1043/0003-

3219(2005)075<0243:ASDEOT>2.0.CO;2 
OWENS, B.M. and KITCHENS, M. The erosive potential of soft drinks on enamel surface substrate: an in vitro scanning electron microscopy investigation. The Journal of Contemporary Dental Practice. 2007, 8(7), 11-20.

SANTIN, G.C., et al. Physical and adhesive properties of dental enamel after radiotherapy and bonding of metal and ceramic brackets. American Journal of Orthodontics and Dentofacial Orthopedics. 2015, 148(2), 283-292. https://doi.org/10.1016/j.ajodo.2015.03.025

SHEIBANINIA, A., et al. The effect of an acidic food-simulating environment on the shear bond strength of self-ligating brackets with different base designs. International Journal of Dentistry. 2014, 2014(6), 689536. https://doi.org/10.1155/2014/689536

TALBOT, T.Q., et al. Effect of argon laser irradiation on shear bond strength of orthodontic brackets: an in vitro study. American Journal of Orthodontics and Dentofacial Orthopedics. 2000, 118(3), 274-279. https://doi.org/10.1067/mod.2000.106069

TÜRKKAHRAMAN, H., et al. In vitro evaluation of shear bond strengths of colour change adhesives. European Journal of Orthodontics. 2010, 32(5), 571-574. https://doi.org/10.1093/ejo/cjp149

TZOU, S. and DARRELL, J. Transbond ${ }^{\text {TM }}$ Plus Color Change adhesive: on-demand convenience with fluoride release, moisture tolerance and color change features. Orthodontics Perspective. 2007, 14(1), 11-13.

WANG, W.N., MENG C.L., TARNG, T.H. Bond strength: a comparison between chemical coated and mechanical interlock bases of ceramic and metal brackets. American Journal of Orthodontics and Dentofacial Orthopedics. 1997, 111(4), 374-381. https://doi.org/10.1016/s0889-

$\underline{5406(97) 80019-4}$

Received: 22 July 2020 | Accepted: 1 September 2020 | Published: 12 January 2021

This is an Open Access article distributed under the terms of the Creative Commons Attribution License, which permits unrestricted use, distribution, and reproduction in any medium, provided the original work is properly cited. 